\title{
Die Plexusbildung der Nerven in der Mittellinie der Rückenhaut einheimischer Frösche.
}

\author{
Von \\ Dr. G. Ottendorff, \\ Assistent am anatomischen Institut zu Bonn. \\ Hierzu 9 Textfiguren.
}

Ueber die Frage, ob die Nerven der Haut bei Menschen und Thieren auch über die Mittellinie hinaus auf die entgegengesetzte Seite übertreten, oder ob die Medianlinie eine unüberschreitbare Grenze bildet, ist von den Anatomen schon viel gestritten worden. So sagt W. Kra u se in seinem Buch über allgemeine und mikroskopische Anatomie: „Jede Nerrenfaser mit Ausnahme des N. opticus endigt auf derjenigen Körperseite, auf welcher sie das Centralorgan verlassen hat (abgesehen von den asymmetrischen Eingeweiden). ${ }^{k}$. $\mathrm{H} \mathrm{enle} \mathrm{drückt} \mathrm{sich} \mathrm{etwas} \mathrm{vor-}$ sichtiger aus: Die peripherischen Nerven überschreiten in den vom Cerebrospinalsystem versorgten Körpertheilen in der Regel nicht die Medianebene. Auch Andere stellen diese Regel mit grösserer oder geringerer Bestimmtheit auf.

Dass diese Behauptung nicht das Richtige treffe, wurde von den Aerzten behauptet. Dr. C. S. Sherrington (Philosophical Transactions of the Royal Society of London 1893) stellte in umfangreichen physiologischen Untersuchungen über die Verbreitung der hinteren Rückenmarkswurzeln unter anderem fest, dass die Empfindungsgebiete der Nerven rentral und dorsal auf kurze Strecken die Mittellinie überschreiten.

Der erste anatomische Nachweis gelang $\mathrm{M}$. $\mathrm{Nussbaum}$ durch Anwendung seiner für die Darstellung von Muskelnerven benutzten Methode auf die Haut des Frosches, der weissen Maus und menschlicher Embryonen. (Ueber den Verlauf und die Endigung peripherer Nerven. Verhandlungen der anatomischen Gesellschaft in Basel 1895.)

Von Z ander wurde die erste bierher gehörige Veröffent- 
lichung 1897 gemacht. (R. Z a n d e r, Ueber das Verhalten der Hautnerven in der Mittellinie des menschlichen Körpers. Sitzungsberichte der biologischen Section der physikalisch-ökonomischen Gesellschaft in Königsberg i. Pr. 28. Januar 1897.) Z a n d e r hat seine Untersuchungen am Menschen gemacht und hat speciell die Kopfnerven genau untersucht. In seiner Schrift: Beiträge zur Kenntniss der Hautnerven des Kopfes, kommt er theilweise auf Grund von Sensibilitätsprüfungen, theilweise durch Präparation der Nerven bis in ihre feinsten makroskopisch darstellbaren Verzweigungen zu dem Ergebniss, dass die Gebiete der verschiedenen Nerven durch Entsendung feiner Aestchen in einander übergreifen, und dass auch die Mittellinie keine Ausnabme hiervon mache. Er führt auch dabei die Untersuchungen von F. Krause aus dem Jahre 1895. (Die Pbysiologie des Trigeminus nach Untersuchungen an Mensehen, bei denen das Ganglion Gasseri entfernt worden ist. Münchener medic. Wochenschrift $1895 \mathrm{Nr} .25,26,27)$ an, der durch Sensibilitätsprtifungen die Grenzen der einzelnen Nervengebiete und ihr Verhalten zur Mittellinie feststellt. Zander hat seine und Krause's Ergebnisse in Kopfschematas sehr übersichtlich eingezeichnet. Daraus geht hervor, dass sich in den meisten Fällen die Erhal: tung der Sensibilität uber die Mittellinie hinaus erstreckt.

Die Arbeiten ron $\mathrm{Z}$ ander datiren in ihren ersten Anfängen aus dem Sommer 1895, zu welcher Zeit er diese Untersuchungen zusammen mit E. F u $\mathrm{k}$ e vornahm, der die Ergebnisse in einer Dissertation zusammengefasst hat.

Die sehr zeitraubende und umständliche Präparation der Nervenfasern, wie sie beim Menschen nöthig ist, erlaubt aber doch nicht die Nerven bis zu ihren Endigungen zu verfolgen. Beim Frosch und auch bei der weissen Maus lässt sich dagegen der Verlauf der Nerven bis in ihre feinsten Verzweigungen auf eine sehr einfache und sichere Art feststellen, wenn man nach der von M. Nussbaum angegebenen Methode verfährt. Das Material zu diesen Untersuchungen verdanke ich Herrn Professor $\mathrm{Nussbaum.} \mathrm{Ein} \mathrm{Theil} \mathrm{der} \mathrm{Vorarbeiten} \mathrm{ist} \mathrm{unter} \mathrm{seiner} \mathrm{Leitung}$ von meinem Vorgänger am hiesigen anatomischen Institut, Herrn Dr. R. Förster, ausgeführt worden, der mir seine Notizen in liebenswürdigster Weise zur Benutzung übergab.

Es beziehen sich diese Untersuchungen speciell auf die 
Rückenhaut des Frosches. Ich will zunächst den makroskopischen Verlauf der betreffenden Nerven schildern und folge dabei dem Werk über die Anatomie des Frosches von A. Eeker und R. W i e d e r s h e im, neubearbeitet von E. G a up p, Freiburg, 2. Aufl. 1897 S. 159.

\section{A. Rami dorsales.}

Die Rami dorsales der Spinalnerven sind bestimmt für die Versorgung der Muskulatur und Haut des Rückens und zeigen in den einzelnen Regionen der Wirbelsäule ein Verbalten, das im Wesentlichen gleichartig ist und nur durch mehr untergeordnete Anpassungen an die specielle Ausbildung der Muskulatur, sowie durch den Mangel der Hautnerven in einzelnen Gebieten eine Modifikation erfährt. Sie treten am dorsalen Umfang der Kalksäckchen hervor; der für den M. intertransversarius : bestimmte Ast meist selbstständig neben oder vor den anderen. Diese letzteren steigen entweder schon getrennt oder noch zusammengefasst dicht vor dem zugehörigen Querfortsatz zwischen diesem und dem Gelenkfortsatz am medialen Rande des M. intertransversarius dorsalwärts und gehen dann auseinander.

\section{a) Rami muscularis.}

Sind bestimmt für die MM. intercrurales und MM. intertransversarii und die Pars dorsomedialis des M. ilio-lumbaris, sowie fur den M. longissimus.

b) Rami cutanei dorsi.

Für die Haut des Rückens bestimmte Aeste kommen nicht allen Spinalnerven zu. Sie fehlen gewöhnlich dem II. und III., sowie dem VIII., IX. und X. Nerven. Vorn ist es der R. auricularis N. vagi, der sein Gebiet bis über die Scapulargegend rïckwärts ausdehnt. Hinten wird die normale Nichtbetheiliguing dreier Spindelnerven an der Versorgung der Rückenhaut ausgeglichen durch die starke Rückwärtsverlängerung des davor gelegenen Hautnerven. Hin und wieder sind auch Rückenhautnerven vom VIII., IX. und X. Spinalnerven, auch einseitig; vorhanden und ebenso findet sich gelegentlich ein solcher Ast rom III. Spinalnerven.

Zwei Reihen von R. cutanei dorsi sind zu unterscheiden, 
eine mediale and eine laterale. Doch kommen beide Aeste zugleich nur den Nn. spinales IV, V, VI, VII zu.

Rami cutanei dorsi mediales.

Die medialen Rückenhautäste des IV. bis VII. Nerven steigen von den Ganglien aus dorsalwärts, dann medialwärts über die zugehörigen MM. intercrurales und treten am medialen Rande des M. longissimus dicht neben der Mittellinie mit caudalwärts gerichtetem Verlauf durch die Fascia dorsalis. Die Durchtrittsstelle durch die Fascie liegt entweder dicht neben dem Dornfortsatz des zu dem Nerven gehörigen Wirbels oder etwas caudal davon. Die $R$. cutanei dorsi mediales ziehen frei durch den dorsalen Lymphsack nach hinten und zur Seite, um erst nach längerem Verlauf an die Haut zu treten. Ihr Gebiet erstreckt sich im Allgemeinen seitwärts bis an das dorsale Septum, dessen Ansatz an der Haut äusserlich durch die vom Augenwinkel rückwärts ziehende verdickte Linie markirt ist. Doch greifen sie auch uber diese Linie hiaus.

In dem binteren Rumpfgebiet kommen ganz besonders viele Varianten in der Verteilung der medialen Rückenhautäste vor. Meist treten seitwärts rom hintersten Steissbeinabschnitt zwei mediale Hautäste durch die Fascia dorsalis, der eine etwas cranial von dem andern. Diese können entstammen: dem $R$. dorsalis $\mathrm{X}$-XI oder dem R. dorsalis XI-XII, oder sie sind Aeste des $R$. dorsalis XI, mit oder ohne Anastomose des binteren Astes mit dem R. dorsalis XII. Ueber die Häufigkeit dieser verschiedenen Vorkommnisse fehlt mir ein Urtheil. Immer verlaufen die beiden Aeste erst eine Strecke subfascial neben dem Steissbein, ehe sie nach aussen abbiegen.

Einige Male beobachtete $G$ a a p p einen R. cutaneus dorsi medialis des dritten Spinalnerven. Er trat seitwärts vom Proc. spin. vert. III durch die Dorsalfascie. Auch ein entsprechender Ast des N. spinalis VIII kommt vor, der des N. IX scheint, wenn er überhaupt vorkommt, besonders selten zu sein.

Sobald die Nerven die Haut erreicht haben, ist ihre Verfolgung mit blossem Auge nicht mehr möglich. Schon $\mathrm{Czermak}^{1}$ ) hat vor vielen Jahren die auch sonst in der histologischen

1) Müller's Archiv 1849, pag. 252 sqq. 
Technik gebräuchliche, stark verdünnte Essigsäure zur Entfernung des Epithels und der äusseren Schichten des Corium beim Studium des Verlaufes der Frosch-Nervenfasern benutzt. Diese alte Methode ist dann von $\mathrm{Nussbaum}$ in folgender Weise verbessert und erweitert worden.

Man löst die Ruickenhaut des Frosches, am geeignetsten sind grössere Exemplare von Rana fusca, in ihrer ganzen Länge und in der Breitenausdehnung zwischen den beiden Rückenwülsten ab und durchschneidet die im Rückenlymphsack verlaufenden Nerven, spannt dann die Haut mit der pigmentirten Fläche nach oben in einer flachen mit Paraffin ausgegossenen Schale auf und ïbergiesst sie mit einer $0,8 \%$ Lösung von Essigsäure. Diese lässt man 3-6 Tage bei circa $15^{\circ}$ Celsius einwirken. Man kann alsdann das Epithel und die äussere stark pigmentirte Schicht des Corium mit Pincetten von der Unterlage abbeben und braucht nur selten mit dem Messer nachzuhelfen, um die Trennung vollständig zu machen. Dann wird das Präparat mit aufwärts gewendeter Innenfläche für 10 Minuten in eine $0,1 \%$ Osmiumsäurelösung gebracht, bis sich die Nerven grau färben. Es folgt darauf eine Auswässerung des Präparates in fliessendem Wasser anf die Dauer von 24 Stunden, wobei sich die Nervenfasern schwärzen. Die weitere Härtung erfolgt in Alkohol von steigender Concentration. Eingeschlossen wird das Präparat in Glycerin. Die Behandlung mit Essigsäure hat den Zweck, die Einwirkung der Osmiumsäure auf das umgebende Gewebe zu verhindern, so dass nur die Nervenfasern intensiv geschwärzit erscheinen.

Es lässt sich an derartigen Präparaten die Ausbreitung der Nerven mit grosser Genauigkeit verfolgen, da alle in der gleichen Ebene auf der Unterfläche des Corium gelegen sind.

Betrachten wir zunächst die Theilung eines Nerven direkt nach seinem Eintritt in die Haut.

In den meisten Fällen theilt sich der Nerv erst nach seinem Eintritt in die Haut; es ist jedoch auch nicht selten, dass die Theilung und zwar in zwei oder auch mehr Aeste schon vor dem Eintritt stattfindet.

Das Grundschema der Theilung ist folgendes (Fig. 1):

Der Nery $(R)$ theilt sich nach seinem Eintritt in die Haut in einen medialen $(R a m)$, einen lateralen $(R a l)$, einen caudalen 
(Raca) und einen cranialen (Rac) Ast. Von diesem Schema kommen zahlreiche Abweichungen vor, doch findet man meistens, wenn der eine oder andere Ast fehlt, dass dieser als secundärer Ast aus einem der benachbarten hervorgeht.

So findet man nach untenstehender Zeichnung, dass der laterale Ast von dem cranialen abgeht, wäbrend die anderen dem Normalschema entsprechen (Fig. 2).

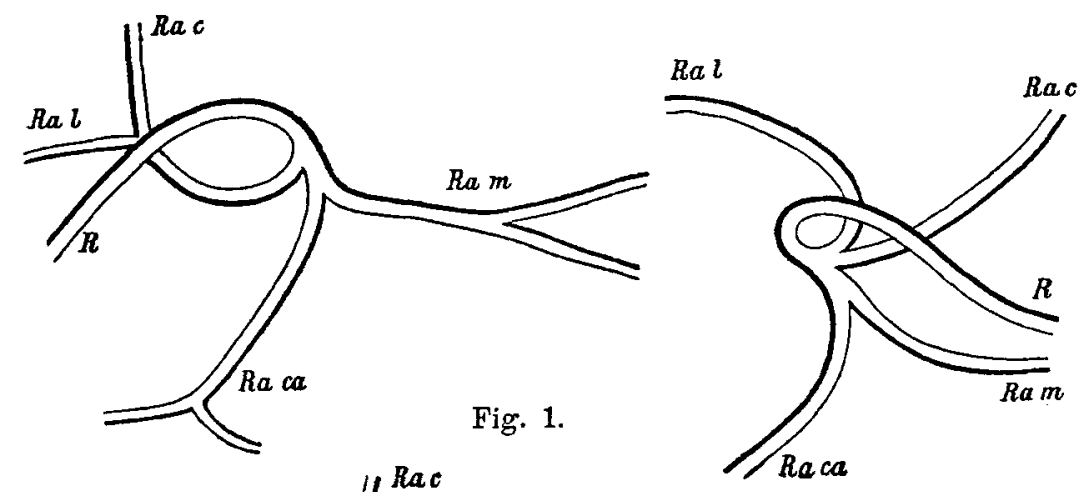

Fig. 2.

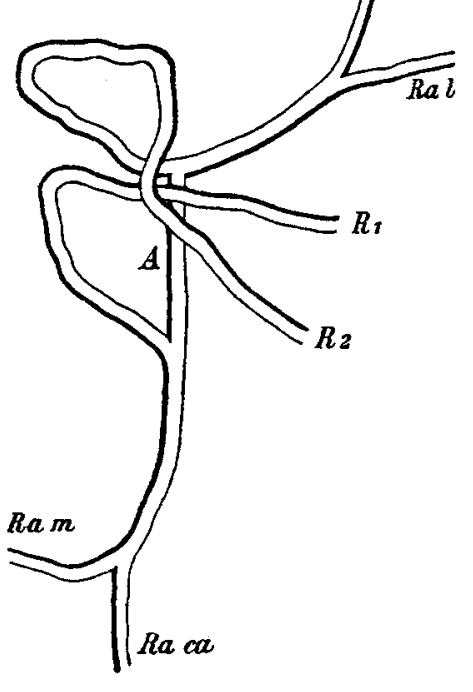

Fig. 3.

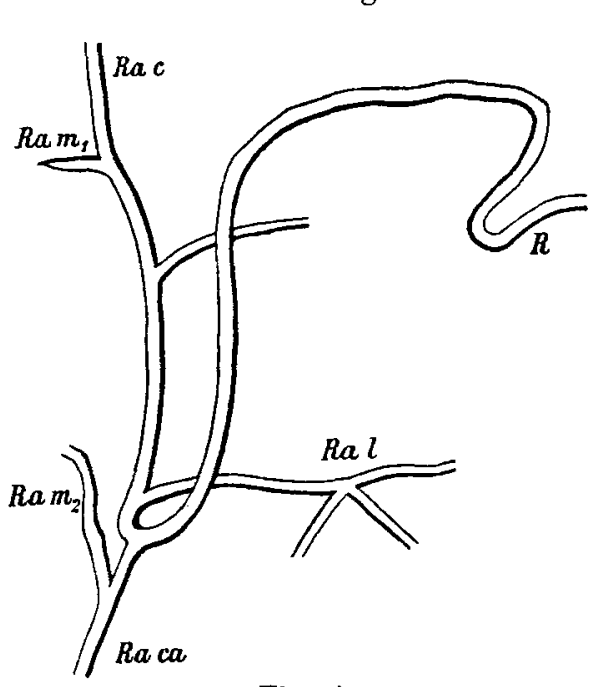

Fig. 4.

Bei Figur 3 theilt sich der Nerv schon vor dem Eintritt in die Haut, wobei der eine Ast $\left(R_{2}\right)$ sich in einen R. lateralis $(R a l)$ und R. cranialis $(R a c)$ theilt, der andere Ast $\left(R_{1}\right)$ in einen 
R. medialis (Ram) und caudalis (Raca). $\quad R_{1}$ und $R_{2}$ sind durch die starke Anastomose $A$ miteinander verbunden.

Bei Zeichnung 4 sind zwei schwächere mediale Aeste $\left(\right.$ Ram $_{1}$ u. $\operatorname{Ram}_{2}$ ) vorhanden, von denen der eine aus dem cranialen, der andere aus dem caudalen Ast hervorgeht.

Im weiteren Verlauf der Nerven herrscht die dichotomische Theilung vor, jedoch findet man vielfach drei- oder auch vierfache Theilungen.

Die Nerven bilden einen reichen Plexus, und zwar finden sich die Anastomosen nicht nur zwischen den Aesten desselben Nerven, sondern auch zwischen den Aesten benachbarter Nerven.

Die umstehende Abbildung (Fig. 5) zeigt die Nervenvertheilung

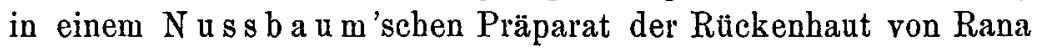
fusca. Man erkennt die Plexusbildung zwischen den zwei N. cutanei dorsi mediales derselben Seite und über die Mittellinic hinüber mit gleichen der anderen Seite. $M o, M c=$ Mittellinie. $M o$ entspricht dem cranialen, $M, c$ dem caudalen Pol, $N=$ Stamm eines $N$. cutaneus dorsi medialis (vergl. Fig. 9).

Die Zeichnung ist auf folgende Weise hergestellt worden. Das betreffende Präparat wurde unter Benutzung eines genan arbeitenden, verschiebbaren Objecttisches in kleine Bezirke eingetheilt und in 42 Einzelphotographien bei einer 54fachen Vergrösserung aufgenommen. Die verschiedenen Photographien wurden sodann zu einer einzigen Tafel vereinigt, die zur Herstellung einer verkleinerten Photographie diente. Von dieser stammt die vorliegende Abbildung als Pause; doch sind nur der Verlauf der Nervenverzweigungen, nicht aber die in der Photographie sichtbaren einzelnen Nervenfasern, die Blutgefässe und Pigmentzellen copirt worden. Die aus dem Plexus in die höheren Coriumschichten aufsteigenden Nerven sind nur rechts unten genau eingetragen. An anderen Stellen sind deren mehr vorhanden, als die Zeichnung anfweist. Die Originalphotographie verdanke ich Hernn Professor $\mathrm{N} \mathrm{u} \mathrm{s} \mathrm{s} \mathrm{b} \mathrm{a} \mathrm{u} \mathrm{m}$, unter dessen Leitung und Beihülfe dieselbe von Herrn cand. \med. S chorlem mer angefertigt wurde (vergl. Sitz.-Ber. der Niederrh. Ges. 1897, pag. 27).

Die Plexusbildungen existiren aber nicht nur, wie die Abbildung zeigt, zwischen Nerven derselben Seite, sondern sie überschreiten auch die Mittellinie in der Art, dass sowohl einander gegenüberliegende als auch aus verschiedenen Rückenmarkswurzeln stammende Nerren sich an der Plexusbildung betheiligen. 
G. Ottendorff:

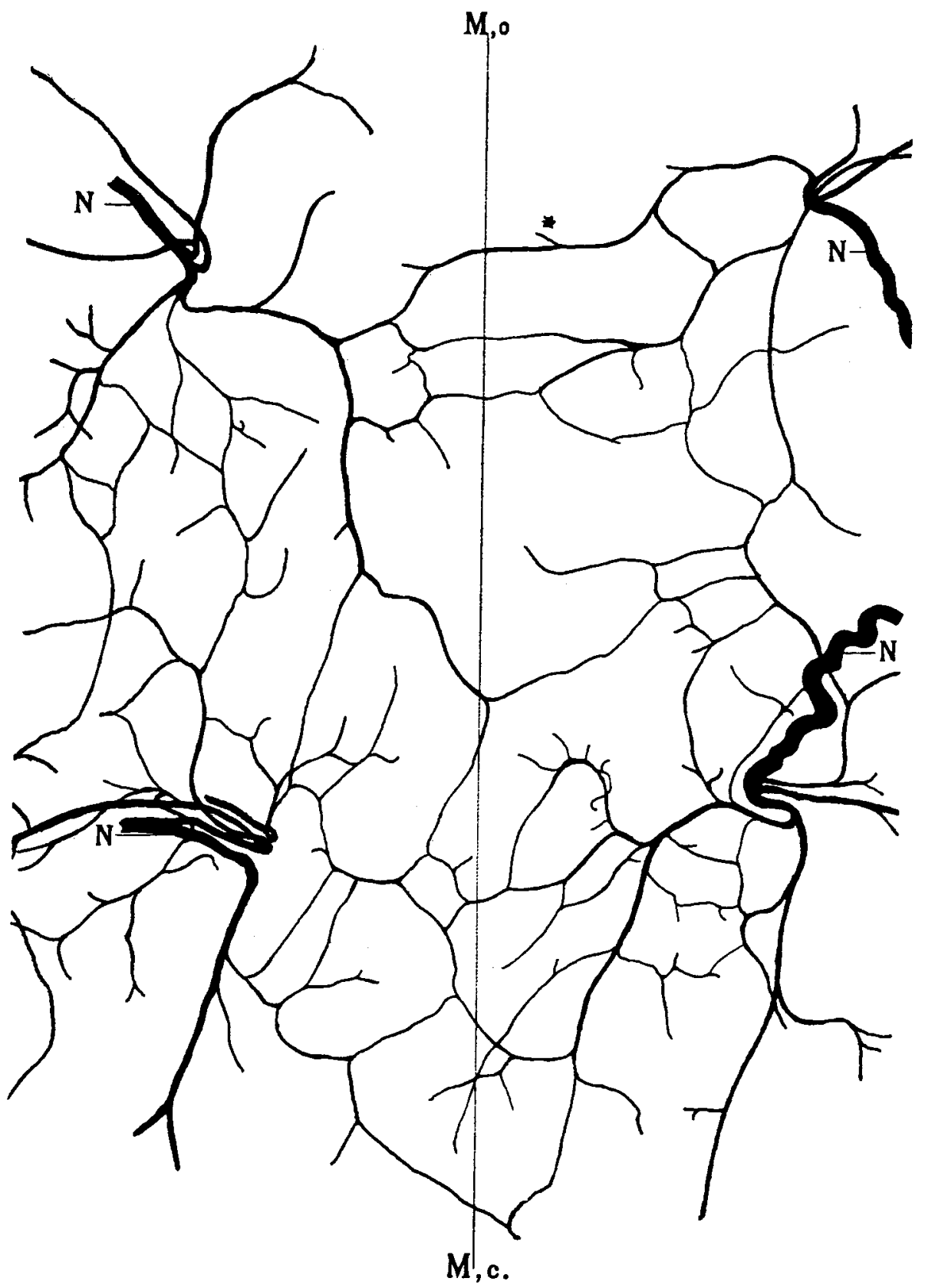

Fig. 5. 
Im weiteren Verlauf der Nervenfasern wird die Plexusbildung viel hänfiger. Man sieht die einzelnen Nervenfasern eines Stammes in das Gebiet der anderen Nervenstämme übergehen, so dass ein Hautbezirk von sehr verschiedenen Stämmen aus innervirt werden kann. Es sind dies aber im Gegensatz zu den von $\mathrm{Z}$ ander für den Menschen ausgesprochenen Behauptungen nicht blos die Grenzgebiete der verschiedenen Nervenstämme, sondern es reichen die Fasern vielfach bis in die Mitte anderer Nervengebiete und auch über die Mittellinie hinaus.

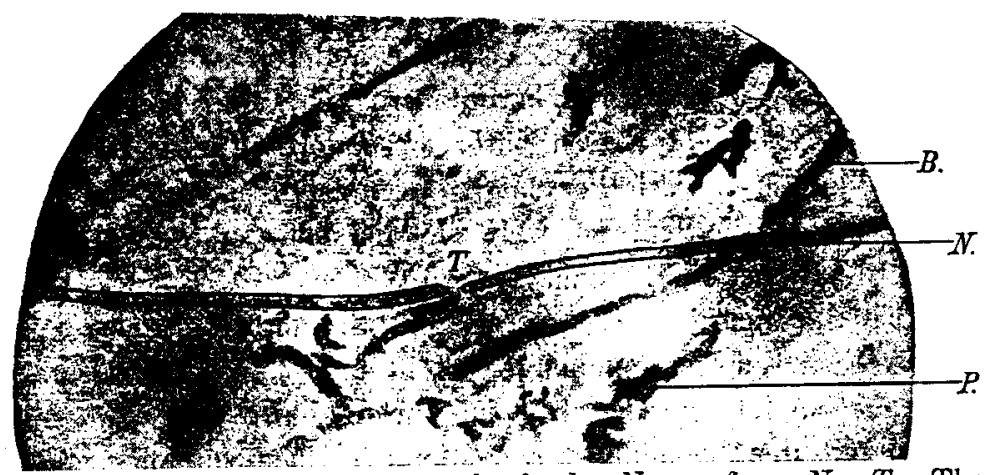

Fig. 6. Theilung einer isolirt verlaufenden Nervenfaser $N . \quad T=$ Theilungsgestelle der Nervenfaser; $B=$ Blutgefässcapillare; $P=$ Pigmentzelle. Nach rechts lässt sich die Faser in den Hauptnervenstamm hinein verfolgen; nach links zieht sie weiter unter Abgabe von Aesten, die wie der bei $T$ gelegene das Corium durchbohren und dann ihr Mark verlieren.

Es handelt sich bei diesen Plexusbildungen nicht allein um Fasern, die zuerst in dem einen Stamm verlaufeu, um dann in den anderen überzugehen. Man findet auch isolirte Nervenfasern, die an verschiedenen Stellen sich theilen und ibre Theiläste entweder anderen Fasern beimischen oder überall isolirt durch die Lederhaut in die Höhe steigen lassen. Die Theilungstellen haben gewöhnlich die Form eines T. Der horizontale Schenkel ist durch eine Ranvier'sche Einschnürung unterbrochen und der verticale ebenso gegen den horizontalen abgesetzt. Der verticale Schenkel ist, wenn die Faser selbst weiter zieht, um sich nochmals zu theilen, der durchbohrende. In den Nervenfasersträngen kommen gewöhnlich Y-förmige Theilungen vor. Ranvier'sche Einschnürungen finden sich auch zwischen den Theilungsstellen im Verlauf der ungetheilten Fasern. Die Theilung einer isolirten markhaltigen Nervenfaser ist obenstehend abgebildet worden. 
An einer anderen Stelle liessen sich ans einer einzigen Nervenfaser zehn durch Theilung derselben entstandene Fasern bis in die höheren Lagen des Coriums verfolgen. Diese einzige Faser versorgt mit ihren Theilstücken ein Gebiet von ca. 2 $\square \mathrm{mm}$.

Besonders ausgezeichnet sind derartig isolirt verlaufende und sich deutlich theilende Nervenfasern durch ihre Dicke und ihre intensive Färbbarkeit in Osmiumsäure.

$\mathrm{Ob}$ ibnen eine besondere Funktion zukommt, und ob sie mit besonderen Endorganen in Verbindung stehen, bedürfte einer besonderen Untersuchung. Soweit sich erkennen liess, durchbohren sie in derselben Weise wie die anderen Nerven die Haut. Der Ursprung derartiger Nervenfasern im Centralnervensystem würde sich vielleicht wegen ibrer besonderen Dicke durch die Wurzeln hindurch verfolgen lassen, wenigstens waren sie in den Stümpfen der Nerven, so weit sie in dem Präparat erhalten waren, deutlich zu erkennen.

Ein bestimmtes Gesetz über die Bildung des Plexus lässt sich nach diesen Untersuchungen nicht aufstellen, doch lässt sich jedenfalls soviel sagen, dass im Durchschnitt jeder Ast eines Nerven mit den benachbarten Aesten desselben Stammes und mit den ihm zugewandten Aesten der benachbarten Stämme ein oder mehrmals in Verbindung tritt, so dass dieselbe Stelle der Haut nicht nur von dem zunächst liegenden, sondern auch von entfernteren Nervenstämmen aus versorgt wird und zwar nicht bloss von den ober- und unterhalb gelegenen Aesten aus, sondern auch mit grosser Häufigkeit über die Mittellinie hinüber.

Die feinere Anordnung der Nervenprimitivfasern bei der Plexusbildung lässt sich nach der oben angegebenen Methode leicht verfolgen. Man findet dabei, dass die Fasern theils in dem ersten Nerven weiter verlaufen, theils in einen andern übergehen, wobei häufig eine Theilung einzelner Nervenprimitivfasern stattfindet. Vielfach findet auch eine Ueberkreuzung der Nervenfasern statt, so dass bei der weiteren Theilung der Nerven Fasern in die Aeste der Nerven eintreten, die ans einem räumlich ziemlich entfernt liegenden Nerven stammen. Es lässt sich ganz genau feststellen, dass häufig Nervenfasern, die ganz nahe bei einander enden, aus zwei verschiedenen Wurzeln derselben oder der entgegengesetzten Seite herstammen.

Ueber derartige Plexus, jedoch ohne Beziehung auf die 
Die Plexusbildung derNerven in der Mittellinie der Rückenhaut etc. 141

Mittellinie, giebt bereits auch $\mathrm{Schw}$ a $\mathrm{lb}$ e in seinem Lehrbuch der Neurologie 1881, pag. 310 an: Es betheiligen sich an solchen Endplexus entweder nur die verschiedenen feinen. Zweige ein und desselben Nerven oder Zweige verschiedener Nerven. In allen Fällen findet der reichlichste Fasernaustausch statt, so dass in Folge dessen ein und dieselbe Körperstelle z. B. ein und dieselbe Strecke der Haut von verschiedenen feinen Nervenzweigen aus Fasern erhalten kann.

Es dürfte jedoch dieser letzte Satz richtiger lauten: ron verscbiedenen feinen Nervenzweigen aus Fasern erbalten kann und auch erhält.

Zum weiteren Beweis derartigen Uebergangs von Nerrenfasern in das Gebiet anderer Nervenstämme und speciell für den Uebertritt von Nervenfasern uber die Mittellinie wurden von Dr. Foerster auf Veranlassung von Profesor $\mathrm{Nussbaum}$ an einer Reihe von Fröschen Operationen ausgeführt, um durch die Degeneration einzelner Nervenstämme die aus anderen Nerven stammenden Fasern desto leichter erkennen zu können.

Diese Versuche dehnten sich vom 25. Mai bis 10. August 1897 aus.

Es wurde die Operation in zweierlei Weise gemacht. Erstens indem auf der Rückenhaut des Frosches durch einen der Mittellinie parallelen Schnitt an der medianen Kante des seitlichen Rückenwulstes der Rückenlymphsack eröffnet wurde. Von den in. dem Rückenlymphsack verlaufenden R. cut. dorsi med. wurden einer. oder mehrere durchschnitten und die Wunde durch Nabt geschlossen. Durch den parallel der Mittellinie verlaufenden Schnitt waren alle Nervenelemente, die von seitwärts in den Lappen treten, zerstört; durch die Zerschneidung der Nervenstämme alle die Fasern, die sich von da aus in dem Hautlappen verbreiten, so dass nur die über die Mittellinie herübertretenden Fasern und die cranialwärts oder caudalwärts binzukommenden erhalten blieben. Um auch diese letzteren auszuschalten, wurde der Längsschnitt durch zwei senkrecht dazu stehende Schnitte, die bis zur Mittellinie reichten, vervollständigt, so dass ein Lappen entstand, der seine Versorgung nur über die Mittellinie berüber erbalten konnte. Es fand sich die Empfindung in den betreffenden Hautpartien kurz nach der Operation in geringem Maasse berabgesetzt; nach Verlauf von 2 bis 3 Monaten aber wieder anscheinend normal. In 
diesem Stadium wurden die Versuchsfrösche dann getödtet und die Rückenhaut in der angegebenen Weise präparirt.

Bei der mikroskopischen Untersuchung ergab sich, dass sich die durchschnittenen Nerven nicht wieder regenerirt hatten. Sie erschienen in den nach oben angegebener Methode hergestellten Präparaten als ganz blasse Stränge ohne bestimmte Differenzirung, während die erbaltenen Fasern sich durch tiefschwarze Färbung auszeichneten. Bekanntlich hat $R$ anvier zuerst den Schwund des Nervenmarks an durchschnittenen Nerven beobachtet (Ranvier, Sur le systeme nerveux. Paris 1878). Es liessen sich daher mit grösster Leichtigkeit die Fisern der erhaltenen Nervenstämme verfolgen und speciell ihr Uebertreten über die Mittellinie. In denjenigen Präparaten, wo noch die Möglichkeit eines Nerveneintritts in die operirte Gegend von der Kopf- oder Schwanzgegend her vorhanden war, fanden sich derartig verlaufende Fasern. Wo diese Möglichkeit durch die Querschnitte der Haut ausgeschlossen worden war, fanden sich nur einige Fasern, die von den Stämmen der unverletzten Seite über die Mittellinie hinaus in die operirte Seite übertraten, während die Hauptmasse der dieser Seite ursprünglich angehörigen Fasern degenerirt war.

Gehen wir za den einzelnen Versuchen über, deren Protokoll mir von Dr. Förster gütigst zur Veröffentlichung überlassen wurde. Ich berichte hierbei nur über die Versuche, die bis $z u$ Ende durchgeführt werden konnten. Eine Reihe von Thieren ging an verschiedenen Erkrankungen vorher zu Grunde.

$$
\text { Versuch I. }
$$

25. Mai Operation: Lappenschnitt links, ein Nerv durchschnitten und zu einer Schleife zusammengebunden, um das Auswachsen und eventuelle Wiederverheilen hintanzuhalten. Hautwunden mit mehreren Seidennähten geschlossen. Erregbarkeit auf dem ganzen Lappen post operationem herabgesetzt.

29. Mai Erregbarkeit im cranialen Theile des Lappens abgeschwächt, im caudalen nur sehr wenig.

2. Juni Erregbarkeit im ganzen wenig herabgesetzt.

18. Juni Erregbarkeit etwas abgeschwächt, besonders im cranialen Theil.

26. Juni Erregbarkeit auf dem ganzen Lappen nur wenig abgeschwächt. Hautwunde verheilt. 
Die Plexusbildung der Nerven in der Mittellinie der Rückenhaut etc. 143

8. Juli Erregbarkeit in dem cranialen Theile noch ein wenig herabgesetzt.

2. Sept. Erregbarkeit auf dem ganzen Lappen gleichmässig vorhanden, vielleicht noch eine Spur herabgesetzt. Getödtet zur Anfertigung des Präparats. Der Nervenstumpf unverändert.

Bei Betrachtung des Präparates, das nach der oben angegebenen Methode angefertigt wurde, zeigte sich, dass der durchschnittene Nerv sich nicht regenerirt hatte. Es war nur das bindegewebige Gerust erhalten, während die Substanz der eigentlichen Nervenfasern ganz verschwunden war. Von dem Nerven der anderen Seite reicht ein starker Ast meist in das Gebiet des degenerirten Nerven hinein; auch einige kleinere Aestchen überschreiten noch die Mittellinie. Von einem Eintritt der Nerven von oben oder unten her ist nichts $z \mathfrak{u}$ bemerken.

\section{Versuch II.}

29. Mai Operation wie bei Versnch I; zwei Nerven durchschnitten. Erregbarkeit post operationem nur im oberen Theile ein wenig herabgesetzt.

4. Juni Erregbarkeit im Ganzen etwas abgeschwächt.

11. Juni Erregbarkeit anscheinend normal.

18. Juni Erregbarkeit vielleicht eine Spur abgeschwächt.

26. Juni nur sehr wenig abgeschwächt. Hautwunde narbig zusammengezogen. gesetzt.

8. Juli Erregbarkeit auf dem ganzen Lappen etwas herab-

31. August Erregbarkeit anscheinend normal. Getödtet zur Anfertigung des Präparates. Auf der linken Seite nach dem Kopfe $z \mathfrak{u}$ ist die Haut auf einer etwa linsengrossen Stelle mit der Fascie verklebt; die Fascie ist daselbst braun verfärbt. Keine neuen Nerven zu sehen; die alten centralen Stümpfe unverändert. Lymphsack sonst gut erhalten.

Die mikroskopische Untersuchung des Präparates ergiebt dasselbe Resultat wie bei dem vorigen. Die beiden durchschnittenen Nerven sind zu bindegewebigen Strängen degenerirt. Ein Uebertreten geschwärzter, also normaler Fasern über die Mittellinie findet in reichem Maasse statt, ron oben und unten her jedoch nicht. 


\section{Versuch III.}

2. Juni Operation: Längsschnitt, links zwei Nerven resecirt. Hautwunde mit fortlaufender Seidennaht geschlossen. Erregbarkeit post operationem anscheinend unverändert.

11. Juni Erregbarkeit unverändert.

8. Juli median vom Hautschnitt ein wenig herabgesetzt.

28. Juli Erregbarkeit anscheinend normal. Getödtet zur Anfertigung des Präparates. Lymphsack gut erhalten, keine Veränderungen zu sehen.

Die mikroskopische Untersuchung zeigt das Uebertreten zahlreicher Nervenfasern in das Gebiet der durchschnittenen Nerven und zwar von oben und unten her and übẻr die Mittellinie herüber; die letzteren gehen theilweise bis beinahe zur Mitte des Gebietes der degenerirten Nerven. An diesen selbst lässt sich keine bestimmte Differenzirung wahrnebmen, nur bindegewebige Stränge und dazwischen eingelagerte schollige Massen, anscheinend Degenerationsprodukte der Nervensubstanz. Dass sie bei den beiden ersten Präparaten fehlten, dürfte wohl daran liegen, dass bei diesen der Zeitraum der Operation und Tödtung länger war, so dass die Resorption der Degenerationsprodukte vollständiger geschehen konnte. Regenerationserscheinungen an den Nerven sind nirgends zu sehen.

\section{Versuch IV.}

18. Juni Operation: Lappenschnitt links. Resection von vier Nerven. Wunde mit fortlaufender Naht gescblossen. Erregbarkeit post operationem im oberen Theile etswas abgeschwächt, unten nur sehr wenig.

26. Juni Erregbarkeit oben nur ganz wenig abgeschwächt. Chromatophoren auf dem Lappen unverändert.

8. Juli Erregbarkeit im oberen Drittel etwas abgeschwächt.

26. Juli Erregbarkeit anscheinend normal. Getödtet zur Anfertigung des Präparates. An der Schnittstelle sind die Hantschichten fest verklebt. Die centralen Nervenstümpfe scheinen etwa $0,5-1 \mathrm{~mm}$ länger geworden $\mathrm{zu}$ sein. Sonst keine Veränderung sichtbar. 
Die Plexusbildung der Nerven in der Mittellinie der Rückenhaut etc. 145

Mikroskopische Untersuchung: Die peripherischen Verzweigungen der vier durchschnittenen Nerven sind degenerirt und zeigen ausser faserigem Bindegewebe an einigen Stellen eingelagerte körnige Massen, die durch die Osmiumsäure geschwärzt sind, anscheinend Produkte fettiger Degeneration. Ueber die Mittellinie treten sehr zahlreiche Nervenfasern hinüber und erstrecken sich ziemlich weit in das Gebiet der durchschnittenen

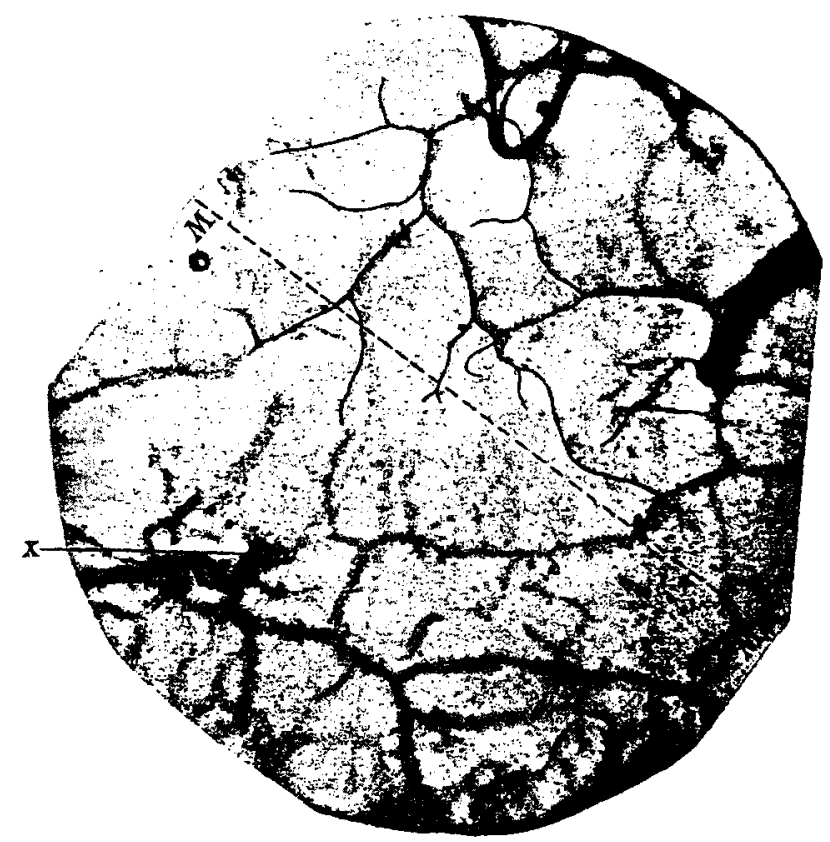

Fig. 7.

Nerven. Von oben und unten her treten keine Fasern ein, da diese durch die Querschnitte bei der Operation zerstört sind.

Aus der Rückenhaut dieses Frosches stammen die beiden Abbildungen 7 u. 8. Fig. $T$ ist nach einer bei schwacher Vergrösserung aufgenommenen Einzelphotographie reproducirt. Das Bild zeigt einen oberhalb der Mittellinie $\left(M M_{1}\right)$ gelegenen Bezirk mit zwei normalen $\mathrm{N}$. cutanei dorsi mediales and eine untere Hälfte mit zwei durchschnittenen und degenerirten gleichnamigen 
Nerren. Der links gelegene Nervenstumpf ist mit $x$ bezeichnet; der rechte tritt nahe an den Bildrand heran und ist nicht mit einem besonderen Buchstaben versehen worden. Die Photographie und besonders die hier benutzte Art der Reproduktion sind nicht im Stande, die Eleganz des Präparates wieder zn geben. Ein Uebelstand besteht darin, dass die degenerirten Nervenstämme (siehe $x$ ), obwohl sie im Osmiumsäurepräparat weiss bleiben, bei durchfallendem Licht undurchsichtig sind und deshalb im photographischen Bilde schwarz erscheinen. Die Reproduktion soll nur die Richtung der Nervenverzweigungen angeben.

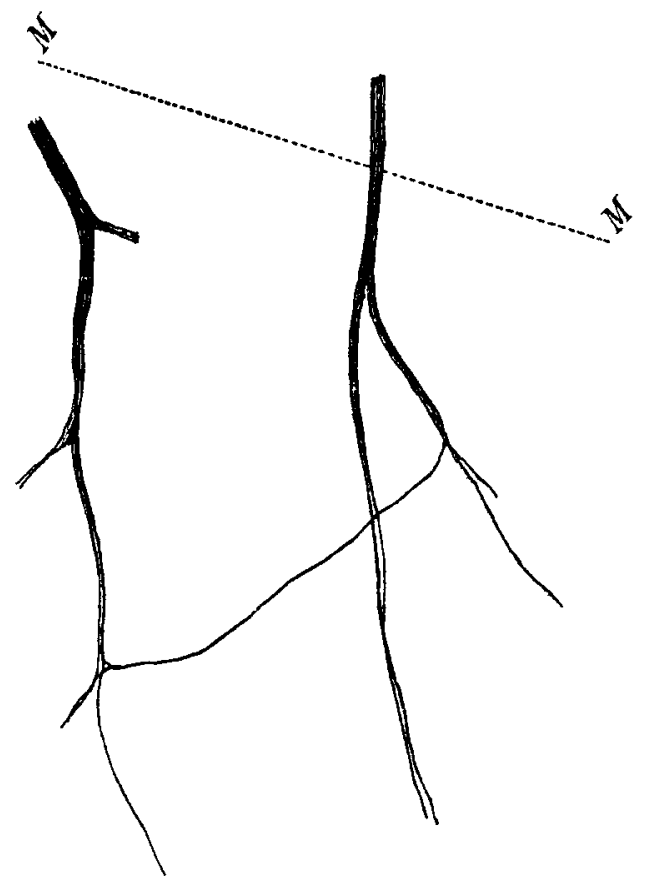

Fig. 8.

In Fig. 8 ist bei Leitz III, Oc. I. ein in die degenerirte untere Hälfte hineinragende, aus der gesunden Seite stammende und deshalb durch die Osmiumsäure geschwärzte Yartie von Nervenfasern abgebildet ( $M M$, Mittellinie). 
Die Plexusbildung der Nerven in der Mittellinie der Rückenhaut etc. 147

\section{Versuch V.}

7. Juli Operation: Lappenschnitt, links zwei Nerven resecirt. Wunde mit fortlaufender Naht geschlossen. Erregbarkeit post operationem im oberen Drittel vielleicht etwas abgeschwächt, sonst normal.

13. Juli Erregbarkeit anscheinend normal.

22. Juli Erregbarkeit im oberen Drittel ein wenig herabgesetzt, ganz unten vielleicht etwas erhöht (?), sonst normal. Getödtet zur Anfertigung des Präparates. Nur die Epidermis lässt sich ablösen, zum Lappen ziehen keine neuen Nerven hin. Gewebe nnter dem Lappen mehr macerirt als auf der gesunden Seite.

Mikroskopische Untersuchung: Die durchschnittenen Nerven sind zu blassen, bindegewebigen Strängen degenerirt; Regenerationserscheinungen sind nicht $z u$ sehen. Der Uebertritt von Nervenfasern aber die Mittellinie ist deutlich zu erkennen; cranial- und caudalwärts treten keine Fasern binzu. Ganz so deutlich wie bei den anderen Präparaten ist das Bild nicht, da sich die Pigment führende Schicht der Haut nur unvollkommen entfernen liess.

Aus diesen Experimenten sowohl, als aus der unter normalen Verhältnissen angestellten anatomischen Untersuchung geht auf das Deutlichste herror, dass die Mittellinie gar kein Hinderniss für den Verlauf der Nervenfasern bildet, dass im Gegentheil der Faseraustausch über die Mittellinie hinüber eher reichlicher ist, als zwischen den Nervenstämmen derselben Seite. Die dadurch bewirkte Innervation ist jedenfalls eine vollkommene; da, wie ja auch der mikroskopische Befund erwarten lässt, die Innervation bei Ausschaltung des einen Nerven vicarïrend durch den anderen übernommen wird, so dass der Ausfall kein ganz vollständiger ist. Ausserdem ist dieser Ausfall nur ein temporärer. Die Versuche zeigten, wie im Verlauf von ein bis zwei Monaten mit Bezug auf Empfindlichkeit restitutio ad integrum eintritt, ohne dass die durchschnittenen Nervenstümpfe sich wieder vereinigt hätten, oder von dem centralen Stumpfe eir neuer Nerv in die operirte Seite der Haut eingetreten wäre.

Zum Schluss füge ich noch Fig. 9 bei, um den Werth der 
Nussbaum'schen Methode für die Verfolgung der einzelnen Nervenfasern in den Plexus zu zeigen.

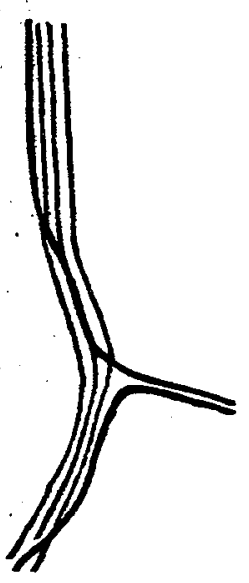

Fig. 9.

Die abgebildete Stelle liegt in dem Präparat, das der Fig. 5 zu Grunde liegt, in Fig. 5 bei *, also nahe der Mittellinie. Die Fig. 9 erläutert somit, wie in dem in der Figur nach rechts unten ans dem Hauptstamm abgehenden durchbohrenden feinen Nervenstamm je eine Faser von oben, das heisst von der einen Körperseite und die zweite von unten her, also von der anderen Körperseite eintritt. Die beiden Nervenfasern des durchbohrenden feinen Nervenstranges würden nach Verlust ihres Markes sich in den höheren Scbichten der Haut verzweigen. Vergr. Leitz, Obj. 5, Oc. II. 